\title{
POTENCIALIDADES ALELOPÁTICAS DEL RESIDUAL PAJA DE LA CAÑA DE AZÚCAR (SACCHARUM SPP., HYBRID) PARA EL MANEJO DE ARVENSES
}

\section{ALLELOPATHIC POTENTIAL OF THE RESIDUAL STRAW OF SUGARCANE (SACCHARUM SPP., HYBRID) FOR WEED MANAGEMENT}

\author{
Walfrido Terrero Matos ${ }^{1}$, Leónides Castellanos González ${ }^{2}$, Liliana \\ Vicet Muro ${ }^{3}$
}

\section{Entidad}

${ }^{1}$ Ing. Agrónomo, Universidad Central de Las Villas. Profesor, Facultad Ciencias Agrarias. Universidad de Cienfuegos Sede "Carlos Rafael Rodríguez", Carretera A Rodas km 4, Cienfuegos, Cuba. Email: wterrero@ucf.edu.cu

${ }^{2}$ Doctor en Ciencias Agrícolas. Universidad Central de Las Villas. , Facultad Ciencias Agrarias. Universidad de Cienfuegos Sede "Carlos Rafael Rodríguez", Carretera A Rodas km 4, Cienfuegos, Cuba. .Email: Iclcastell@gmail.com, http://orcid.org/0000-0001-9285-4879

${ }^{3}$ Dr. En Ciencias Químicas. Universidad Central de Las Villas Profesor Facultad de Química- Farmacia. Universidad Central de las Villas "Martha Abreu", Carretera a Camajuaní Km 10, Santa Clara, Cuba. Email. Ivicet@uclv.edu.cu

\begin{abstract}
Resumen
Para contribuir al conocimiento científico sobre los aleloquímicos presentes en un híbrido de caña de azúcar (Saccharum spp., hybrid), se realizó un trabajo que tuvo como objetivo determinar los metabolitos secundarios que pudieran tener potencialidades alelopáticas contra las arvenses. Se realizó una extracción sucesiva con solventes de polaridad ascendente (éter etílico, etanol y agua), posteriormente, se procedió a la identificación del tipo cualitativo, haciendo uso de reactivos de coloración y precipitación. Para ello se se partió de $5 \mathrm{~g}$ de material vegetal en polvo y se realizó la extracción con $50 \mathrm{~mL}$ de las soluciones, por maceración con agitación constante en una zaranda marca Thys 2 por 24 horas. Luego se filtró para la obtención de los extractos correspondientes. Se les aplicó la técnica de tamizaje fitoquímico establecida por el MINSAP. Se encontró la presencia de resinas, glicósidos cardiotónicos, fenoles y/o taninos, flavenoides, triterpenos, cumarinas y quinonas en el extracto etanólico; ácidos grasos en el extracto etéreo; saponinas, aminoácidos libres, azúcares reductores, flavenoides, fenoles y/o taninos en extracto acuoso. Se considera que los fenoles y taninos presentes pudieran tener efecto alelopático contra las arvenses por lo que la paja de la caña de azúcar resulta un buen candidato para el manejo de malezas por su potencial como herbicida.
\end{abstract}

Palabras clave

tamizaje fitoquímico, alelopatía, metabolitos secundarios, fenoles, taninos

Abstract

Para contribuir al conocimiento científico sobre los aleloquímicos presentes en 
un híbrido de caña de azúcar (Saccharum spp., hybrid), se realizó un trabajo que tuvo como objetivo determinar los metabolitos secundarios que pudieran tener potencialidades alelopáticas contra las arvenses. Se realizó una extracción sucesiva con solventes de polaridad ascendente (éter etílico, etanol y agua), posteriormente, se procedió a la identificación del tipo cualitativo, haciendo uso de reactivos de coloración y precipitación. Para ello se se partió de $5 \mathrm{~g}$ de material vegetal en polvo y se realizó la extracción con $50 \mathrm{~mL}$ de las soluciones, por maceración con agitación constante en una zaranda marca Thys 2 por 24 horas. Luego se filtró para la obtención de los extractos correspondientes. Se les aplicó la técnica de tamizaje fitoquímico establecida por el MINSAP. Se encontró la presencia de resinas, glicósidos cardiotónicos, fenoles y/o taninos, flavenoides, triterpenos, cumarinas y quinonas en el extracto etanólico; ácidos grasos en el extracto etéreo; saponinas, aminoácidos libres, azúcares reductores, flavenoides, fenoles y/o taninos en extracto acuoso. Se considera que los fenoles y taninos presentes pudieran tener efecto alelopático contra las arvenses por lo que la paja de la caña de azúcar resulta un buen candidato para el manejo de malezas por su potencial como herbicida.

Keywords: phytochemical screening, allelopathy, secondary metabolites, tannins, phenols

\section{INTRODUCCIÓN}

La caña de azúcar (Saccharum spp., híbrido) en su estado natural está formada por $8,44 \%$ de cogollo y hojas verdes, $19,74 \%$ de vainas y hojas secas, y $71,82 \%$ de tallos limpios. La paja posee una estructura muy diferente al cogollo, está constituida por la vaina y la hoja seca, mientras que el cogollo contiene tallo y hojas verdes (Bambanaste, 1986).

Sobre este residuo, se plantea que cuando queda sobre el suelo en forma de una cubierta protectora realiza una importante función de conservación de la humedad, evita la erosión y contribuye a la lucha contra arvenses (Rodríguez et al., 2014; Meneses et al, 2016), pero poco se ha informado de su potencial alelopático.

Una forma de utilizar la alelopatía en la agricultura es a través del uso de productos naturales como bioherbicidas. Al respecto Xuan, Tsuzuki, Eumatsu y Terao (2002) afirman que este enfoque al manejo de las arvenses es seguro para el ambiente comparado con los herbicidas sintéticos. También Macías (1995) sugiere el empleo de aleloquímicos naturales o modificados como herbicidas, transferencia genética de propiedades alelopáticas a cultivares comerciales; empleando plantas alelopáticas en rotación de cultivos, ya sea como plantas acompañantes y como abono verde; uso de "mulchings" fitotóxicos y manejo de cubiertas vegetales para la supresión de arvenses (Gómez et al, 2017).

Para contribuir al conocimiento científico sobre los aleloquímicos presentes en la paja de la caña de azúcar, se realizó el presente trabajo que tuvo como objetivo los metabolitos secundarios que pudieran tener potencialidades alelopáticas contra las arvenses (Moreno \& Rueda, 2016).

\section{METODOLOGIA}

El material vegetal fue colectado en la Estación Territorial de Investigaciones de la Caña de Azúcar en el municipio de Ranchuelo, Provincia Villa Clara, en febrero de 2017, para ello se seleccionó la variedad C86-12 por ser líder en Cuba y una de la más plantada en la provincia de Cienfuegos (González et al., 2010). La muestra se trasladó en saco de nylon y se colocó a la estufa a $30{ }^{\circ} \mathrm{C}$ hasta lograr peso constante y quedar crujiente, posteriormente se pulverizó en molino de 
cuchillas (IKA, Chisty y Norris) y se pasó por un tamiz de $1 \mathrm{~mm}$ de diámetro. Al polvo obtenido se le realizó el tamizaje fitoquímico en la Facultad de QuímicaFarmacia de la Universidad Central de las Villa. Para ello, se partió de 5 gramos de material vegetal en polvo de la paja de la caña de azúcar y se realizó una extracción con $50 \mathrm{~mL}$ de éter etílico a temperatura ambiente, por maceración con agitación constante en una zaranda marca Thys 2 , por 24 horas. Posteriormente se filtró con papel de filtro Whatman 1 obteniéndose el extracto etéreo.

El residuo vegetal se secó al aire libre y se le hizo una extracción con $50 \mathrm{~mL}$ de etanol a $98 \%$, siguiendo el procedimiento anterior y se filtró obteniéndose el extracto etanólico.

El residuo vegetal se volvió a secar al aire libre y se realizó otra extracción con $50 \mathrm{~mL}$ de agua destilada obteniéndose el extracto acuoso. Se empleó el procedimiento por maceración descrito anteriormente.

Luego se tomaron alícuotas de $1 \mathrm{~mL}$ de cada extracto y se les aplicó la técnica de tamizaje fitoquímico establecida por el MINSAP, según correspondiera de acuerdo al tipo se disolvente de extracción:

Extracto etéreo: Ensayo de Dragendorff (alcaloides), ensayo de Sudán III (grasos), ensayo de Baljet (cumarinas), ensayo de Liebermann- Burchard (triterpenos y esteroides).

Extracto etanólico: Ensayo de resinas (resinas), ensayo de espuma (aponinas), ensayo de Dragendorff (alcaloides), ensayo de Kedde (glicósidos cardiotónicos), ensayo de Cloruro férrico (fenoles y/o taninos), ensayo de Shinoda (flavonoides), ensayo de LiebermannBurchard (triterpenos y/o esteroides), ensayo de Nihidrina (aminoácidos libres), ensayo de Baljet (cumarinas), ensayo de Fehling (azúcares reductores), ensayo de Borntrager (quinonas).
Extracto acuoso: Ensayo de espuma (saponinas), ensayo de Shinoda (flavonoides), ensayo de Nihidrina (aminoácidos libres), ensayo de Fehling (azúcares reductores), ensayo de Cloruro Férrico (fenoles y/o taninos), ensayo de enfriamiento (mucílagos).

Los resultados se valoraron estableciendo varias categorías de acuerdo a la evidencia en las reacciones: $(-)$ ausencia del metabolito, (+) presencia del metabolito en bajas concentraciones, $(++)$ presencia del metabolito en concentraciones moderadas, (+++) presencia del metabolito en altas concentraciones. Se procedió a la identificación del tipo cualitativo, haciendo uso de reactivos de coloración y precipitación.

\section{ANALISIS $Y$ DISCUSIÓN DE RESULTADOS}

En el extracto etéreo se encontró la presencia de ácidos grasos en bajas concentraciones, en este caso aparecieron pequeñas gotas de color rojo los cuales demostraban un resultado positivo al ensayo. Este metabolito detectado no se considera de interés para su uso como herbicida (tabla 1).

En el extracto etanólico se encontró la presencia de resinas, glicósidos cardiotónicos, fenoles y/o taninos, flavonoides, triterpenos y cumarinas (tabla 2). Se consideró positiva la presencia de resinas en bajas concentraciones por la aparición de una ligera turbidez, así como los glicósidos cardiotónicos por haber tomado una ligera coloración violácea y la aparición de un precipitado carmelita el cual se pudo comprobar que era soluble en agua. 
Tabla 1. Presencia de metabolitos en el tamizaje fitoquímico realizado en extracto etéreo

\begin{tabular}{lccc}
\hline Material Vegetal & Metabolito & Ensayo realizado & Resultados \\
\hline \multirow{4}{*}{ Paja de caña } & Alcaloides & Dragendorff & - \\
& Ácidos grasos & Sudán III & + \\
& Cumarinas & Baljet & - \\
& Triterpenos y esteroides & Liebermann- Burchard & - \\
\hline
\end{tabular}

Fuente: Elaboración propia

Tabla 2. Presencia de metabolitos en el tamizaje fitoquímico realizado en extracto etanólico

\begin{tabular}{cllc}
\multicolumn{1}{c}{$\begin{array}{c}\text { Tabla 2. Presencia de metabolitos en el tamizaje fitoquímico realizado en extracto etanólic } \\
\text { Megetal }\end{array}$} & \multicolumn{1}{c}{ Metabolitos } & Ensayo realizado & Resultados \\
\hline & Resinas & Resinas & + \\
& Saponinas & Espuma & - \\
& Alcaloides & Dragendorff & - \\
& Glicósidos cardiotónicos & Kedde & + \\
& Fenoles y/o taninos & Cloruro férrico & + \\
Paja de caña & Flavonoides & Shinoda & + \\
& Triterpenos y/o & Liebermann- Burchard & - \\
& esteroides & Nihidrina & + \\
& Aminoácidos libres & Baljet & - \\
& Cumarinas & Fehling & + \\
& Azúcares reductores & Borntrager & \\
& Quinonas & &
\end{tabular}

Fuente: Elaboración propia

Tabla 3. Presencia de metabolitos en el tamizaje fitoquímico realizado en extracto acuoso.

\begin{tabular}{lccc}
\hline Material Vegetal & Metabolitos & Ensayo realizado & Resultados \\
\hline & Saponinas & Espuma & + \\
& Flavonoides & Shinoda & + \\
Paja de caña & Aminoácidos libres & Nihidrina & +++ \\
& Azúcares reductores & Fehling & + \\
& Fenoles y/o taninos & Cloruro férrico & +++ \\
& Mucílagos & Enfriamiento & - \\
\hline
\end{tabular}

Fuente: Elaboración propia

Por su parte los fenoles y/o taninos también se detectaron en bajas concentraciones por la aparición de una coloración verde, propia de taninos del tipo pirocatecólicos.

En el caso de los flavonoides se visualizó una ligera coloración rosada por lo que también se consideró positivo en bajas concentraciones.

Aparentemente, las cumarinas y varios flavonoides tienen actividad antagónica contra el efecto inhibitorio del ABA y en ocasiones pueden llegar a estimular así, el crecimiento inducido por el ácido giberélico. La inhibición de crecimiento de plántulas de pepino debida al ácido ferúlico y otros compuestos fenólicos ha sido correlacionada con el incremento en los niveles del ácido abscísico (Blanco, 2006).

Los triterpenos $\mathrm{y} / \mathrm{o}$ esteroides se detectaron en bajas concentraciones por la observación de dos fases: (fase inferior rosado ligero a naranja y la fase superior amarillo pálida). 
Algunos terpenoides son especialmente inhibidores de la fotosíntesis, como los diterpenos labdane-8a y 15-diol aislados de Croton ciliatoglandulifer Ortega (Euphorbiaceae), los que interfieren en la síntesis de ATP, interrumpiendo la transferencia de electrones desde el PS 680 (II) a la plastoquinona B. Con efectos similares, están los sesquiterpenos de hojas de Celastrus vulcanicola Donn. Sm. (Celastraceae) (Torres, King, Jiménez, Lotina y Bozzocchi, 2008).

Por otra parte, se detectaron cumarinas en concentraciones moderadas por la aparición de un precipitado carmelita abundante y se comprobó que este era soluble en agua (Rodríguez, 2017).

Un sitio de acción importante de los aleloquímicos son las membranas celulares, donde pueden producir peroxidación de los lípidos y ruptura de estas. Tales efectos se han observado con la sarmentina, amida extraída de frutos de Piper spp., cumarinas, y fenoles simples como el ácido p-hidroxibenzóico (Dayan, Owens, Watson, Asolkar, y Boddy, 2015).

Otros resultados evidencian actividad de las cumarinas en el movimiento polar de la auxina en mutantes de Arabidopsis thaliana (Lupini, Araniti, Sunseri, y Abenovoli, 2014).

Las quinonas también se consideraron presentes en bajas concentraciones por aparecer una coloración rosada en la parte superior del tubo de ensayo.

En el extracto acuoso se encontró la presencia de saponinas, aminoácidos libres, flavonoides, azúcares reductores, fenoles y/o taninos (tabla 3).

La presencia de algunos metabolitos como saponinas, los flavonoides y azúcares reductores se consideraron positivas en bajas concentraciones, los primeros por la presencia de espuma en la superficie del líquido (de más de $2 \mathrm{~mm}$ de espesor y persiste por más de 2 min), los segundos por la aparición de una ligera coloración rosada y los terceros por que aparece una coloración verde y posteriormente ocurre un precipitado rojo.

Sin embargo, los aminoácidos libres, así como los fenoles y/o taninos arrojaron estar presentes en altas concentraciones, los primeros por la aparición de una coloración violácea intensa y los segundos por la aparición de una coloración rojo vino.

Compuestos fenólicos como taninos, ácidos fenólicos (ferúlico, $p$-cumárico, vainíllico) y cumarinas inhiben la acción de la giberelina en la germinación, al producir precipitación de hidrolasas, principalmente la a-amilasa y fosfatasas ácidas. Paralelamente, las cumarinas pueden reducir el crecimiento primario de la radícula en la germinación y bloquear los sitios de acción de giberelinas (Saleh, Madany y González, 2014).

Los compuestos fenólicos, en particular, los flavonoides actúan en las AIA oxidasas. Los monofenoles (ácidos $p$ hidroxibenzoico, vainíllico, $p$-cumárico y siríngico) reducen la disponibilidad de AIA al promover su descarboxilación. En contraste, muchos difenoles y polifenoles (ácidos clorogénico, caféico, ferúlico y protocatécuico) inhiben las AIA-oxidasas y por tanto, sinergizan el crecimiento inducido por AIA, suprimiendo la degradación de la hormona. Esto sugiere un control en los niveles de AIA a través de la enzima polifenoloxidasa (sintetiza polifenoles) que regula el balance entre monofenoles y polifenoles (Blanco, 2006). La presencia en dos de los extractos de flavenoides y triterpenos y/o esteroides en uno indica que la paja de la caña de azúcar pudiera ser usada por su potencial alelopático en el control de arvenses.

Las cumarinas a pesar de haberse encontrado en un solo extracto (etanólico), 
y en concentraciones moderadas pudieran apoyar el empleo de la paja con el fin de controlar arvenses potenciando el efecto alelopático de los anteriores metabolitos. Los fenoles y/o taninos pudieran tener efecto alelopático contra las arvenses por estar presentes o haberse detectado en dos extractos (etanólico y acuoso), en este último en altas concentraciones

\section{CONCLUSIONES}

El tamizaje fitoquímico realizado a la paja de caña de azúcar reveló la presencia de metabolitos que pudieran ejercer acción alelopáticas contra arvenses. Los fenoles y taninos presentes pudieran tener efecto alelopático contra las arvenses por lo que la paja de la caña de azúcar resulta un buen candidato para el manejo de malezas por su potencial efecto herbicida, por lo que deben realizarse las investigaciones sobre el efecto de este residuo sobre arvenses en laboratorio y campo.

\section{Referencias Bibliografícas}

Angulo, W. J., Mendoza, J. A. y Uriel, H. U. (2017). Análisis de la vulnerabilidad por fenómenos de remoción en masa en la Cuenca Tanauca estudio de caso. Revista Ambiental Agua, Aire y Suelo. ISSN 1900-9178. Volumen (8), Numero

DOI: https://doi.org/10.24054/1900 9178.v2.n2.2017.3276

Aremu, A. O., Masondo, N. A., Rengasamy, K. R., Amoo, S.O., Gruz, J., Bíba, O., \& Van Staden, J. 2015. Physiological role of phenolic biostimulants isolated from brown seaweed Ecklonia maxima on plant growth and development. Plantar, 241(6), 1313-1324.

Bambanaste, R. 1986. El bagazo. Capitulo IV. La industria de los derivados de la caña de azúcar.
Editorial Científico Técnica. La Habana, Cuba. pp.121-130.

Blanco, Y. 2006. La utilización de la alelopatía y sus efectos en diferentes cultivos agrícolas. Cultivos Tropicales, 27(3), 5-16.

Camargo, W. C. (2016). Modelación hidrologico-hidraulica de eventos de inundacion en el Rio Bogotá (sector tocanzipa-chia) usando HEC-RAS. Revista Ambiental Agua, Aire y Suelo. ISSN 19009178. Volumen (7), Numero (2). DOI: https://doi.org/10.24054/1900 9178.v2.n2.2016.3267

Dayan, F. E, Owens, D. K., Watson, S. B., Asolkar, R, \& Boddy, L. 2015. Sarmentine, a natural herbicide from Piper species with multiple herbicide mechanisms of action. Frontiers in Plant Science, 6, 222259.

González, M. G., Almeida, R., Alfonso, F., Pardo, L., Barroso, G., González, J. R, Rodríguez, M. 2010. Principales variedades de Caña de Azúcar propagadas en Cuba al cierre del 2008. Revista de la Asociación de Técnicos Azucareros de Cuba., 71(1): 1925.

Gómez, S. A., Mora, W. J. y Ramón, B. (2017). Caracterización física y química de la fibra natural de esparto (juncus ramboi subsp. colombianus) como alternativa de refuerzo en materiales compuestos. Revista Ambiental Agua, Aire y Suelo. ISSN 19009178. Volumen (8), Numero (1). DOI: https://doi.org/10.24054/1900 9178.v1.n1.2017.3273

Gutierrez, T., Castellanos, C. y Hernández, N. (2016). El ordenamiento territorial frente a las consecuencias de los cambios climáticos. Revista Ambiental Agua, Aire y Suelo. ISSN 19009178. Volumen (7), Numero (2). 
DOI: https://doi.org/10.24054/1900 9178.v2.n2.2016.3338

Lupini, A., Araniti, F., Sunseri, F., \& Abenavoli, M. R. 2014. Coumarin interacts with auxin polar transport to modify root system architecture in Arabidopsis thaliana. Plant Growth Regulation, 74(1), 23-31.

Melo, J., Saavedra, S. y Ramón, J. A. (2017). Evaluación de la adsorcion de $\mathrm{CU}+2$ y azul de metileno en biosorbentes de bajo costo obtenidos a partir de biomasa residual de la agroindustria de cítricos. Revista Ambiental Agua, Aire y Suelo. ISSN 1900-9178. Volumen (8), Numero (2). DOI: https://doi.org/10.24054/1900 9178.v2.n2.2017.3277

Meneses, V. B., Álzate, D. y Mosquera, J. (2016). Sistema de optimización de las técnicas de planificación en agricultura de precisión por medio de drones. Revista Ambiental Agua, Aire y Suelo. ISSN 19009178. Volumen (7), Numero (2). DOI: https://doi.org/10.24054/1900 9178.v2.n2.2016.3268

Moreno, C. y Rueda, L. (2016). La educación ambiental como herramienta para la recuperación de la cobertura vegetal, mediante prácticas agro-ecológicas en la comunidad minera asograstorres, asociación de gravilleros de Sabana de Torres. Revista Ambiental Agua, Aire y Suelo. ISSN 1900-9178. Volumen (7), Numero

DOI: https://doi.org/10.24054/1900 9178.v1.n1.2016.3260

Rodríguez, T. M., Castellanos, L., \& Rodríguez, I. 2014. Manejo de plagas para la caña de azúcar en la Empresa Azucarera "Melanio Hernández". Centro Agrícola, 41(3), 35-41.

Rodríguez, Y. A. (2017). Conservación de humedales en el marco de gestión de cuencas hidrográficas. Puerto Rondón-Arauca. Revista Ambiental Agua, Aire y Suelo. ISSN 1900-9178. Volumen (8), Numero

\section{8.v2.n2.2017.3281}

